\title{
Erratum to: Cyclosporine A vs. methylprednisolone for Henoch-Schönlein nephritis: a randomized trial
}

\author{
Outi Jauhola • Jaana Ronkainen • Helena Autio-Harmainen • Olli Koskimies • \\ Marja Ala-Houhala • Pekka Arikoski - Tuula Hölttä • Timo Jahnukainen • \\ Jukka Rajantie • Timo Örmälä • Matti Nuutinen
}

Published online: 4 August 2011

(C) IPNA 2011

Erratum to: Pediatric Nephrology

DOI 10.1007/s00467-011-1919-5

Unfortunately Table 1 was printed incorrectly. The correct version can be seen below.

The online version of the original article can be found at http://dx.doi. org/10.1007/s00467-011-1919-5.

O. Jauhola $\cdot$ M. Nuutinen $(\triangle)$

Department of Children and Adolescents, Oulu University

Hospital,

PO Box 23, 90029, Oulu, Finland

e-mail: matti.nuutinen@ppshp.fi

O. Jauhola

e-mail: ojauhola@paju.oulu.fi

J. Ronkainen

Oulu City Health Care Centre,

Oulu, Finland

M. Ala-Houhala

Department of Pediatrics, Tampere University Hospital,

Tampere, Finland

P. Arikoski

Department of Pediatrics, Kuopio University Hospital,

Kuopio, Finland

H. Autio-Harmainen

Department of Pathology, Oulu University Hospital,

Oulu, Finland

\section{J. Rajantie}

Department of Pediatrics, Helsinki University Central Hospital, Jorvi Hospital,

Espoo, Finland

O. Koskimies · T. Hölttä • T. Jahnukainen

T. Örmälä

Hospital for Children and Adolescents, University of Helsinki,

Helsinki, Finland

Department of Pediatrics, Hyvinkää Hospital,

Hyvinkää, Finland 
Table 1 Demographic and clinical features of 24 patients with HSN at onset by treatment group and randomization

\begin{tabular}{|c|c|c|c|c|}
\hline & \multicolumn{2}{|l|}{ CyA $(n=11)$} & \multicolumn{2}{|l|}{$\mathrm{MP}(n=13)$} \\
\hline & $\begin{array}{l}\text { Randomized } \\
(n=7)\end{array}$ & $\begin{array}{l}\text { Non-randomized } \\
(n=4)\end{array}$ & $\begin{array}{l}\text { Randomized } \\
(n=8)\end{array}$ & $\begin{array}{l}\text { Non-randomized } \\
(n=5)\end{array}$ \\
\hline Gender & 2 girls, 5 boys & 1 girl, 3 boys & 2 girls, 6 boys & 4 girls, 1 boy \\
\hline \multicolumn{5}{|c|}{ Age at onset (years) } \\
\hline Mean & 9.0 & 10.4 & 7.9 & 11.5 \\
\hline Range & $5.1-12.3$ & $7.0-14.4$ & $4.0-14.8$ & $7.8-16.8$ \\
\hline \multicolumn{5}{|c|}{ dU-Prot $\left(\mathrm{mg} / \mathrm{m}^{2} / \mathrm{h}\right)$} \\
\hline Mean & 143 & 73 & 182 & 128 \\
\hline Range & $38-510$ & $14-229$ & $7-356$ & $83-181$ \\
\hline \multicolumn{5}{|l|}{ SAlb (g/l) } \\
\hline Mean & 33 & 31 & 26 & 27 \\
\hline Range & $20-43$ & $16-40$ & $15-37$ & $14-35$ \\
\hline Hematuria N/\% & $7 / 7(100 \%)$ & $4 / 4(100 \%)$ & $8 / 8(100 \%)$ & $4 / 4(100 \%)$ \\
\hline \multicolumn{5}{|c|}{$\begin{array}{l}\text { Time from HSP diagnosis } \\
\text { to enrollment (months) }\end{array}$} \\
\hline Mean & 3.0 & 9.2 & 3.6 & 4.6 \\
\hline Range & $0.8-11.8$ & $1.7-30.6$ & $0.9-7.8$ & $1.5-8.5$ \\
\hline ISKDC II & 2 & 0 & 3 & 0 \\
\hline ISKDC III & 5 & 4 & 5 & 5 \\
\hline
\end{tabular}

